\title{
ANTIPODAL GRAPHS AND DIGRAPHS
}

\section{GARRY JOHNS and KAREN SLENO}

Department of Mathematical Sciences

Saginaw Valley State University

University Center, Michigan 48710

(Received May 7, 1991 and in revised form November 5, 1991)

\begin{abstract}
The antipodal graph of a graph $G$, denoted by $A(G)$, has the same vertex set as $\boldsymbol{G}$ with an edge joining vertices $u$ and $v$ if $d(u, v)$ is equal to the diameter of $\boldsymbol{G}$. (If $\boldsymbol{G}$ is disconnected, then $\operatorname{diam} G=\infty$.) This definition is extended to a digraph $D$ where the arc $(u, v)$ is included in $A(D)$ if $d(u, v)$ is the diameter of $D$. It is shown that a digraph $D$ is an antipodal digraph if and only if $D$ is the antipodal digraph of its complement. This generalizes a known characterization for antipodal graphs and provides an improved proof. Examples and properties of antipodal digraphs are given. A digraph $D$ is self-antipodal if $A(D)$ is isomorphic to $D$. Several characteristics of a self-antipodal digraph $D$ are given including sharp upper and lower bounds on the size of $D$. Similar results are given for self-antipodal graphs.
\end{abstract}

KEY WORDS AND PHRASES. Antipodal graphs, Antipodal digraphs. 1991 AMS SUBJECT CLASSIFICATION CODES. 05C12, 05C20.

\section{INTRODUCTION.}

We will use Chartrand and Lesniak [1] for basic terminology and notation.

For a pair $u, v$ of vertices in a strong digraph $D$ the distance $d(u, v)$ is the length of a shortest directed $u-v$ path. We can extend this definition to all digraphs $D$ by defining $d(u, v)=\infty$ if there is no directed $u-v$ path in $D$. Similarly, if $G$ is a disconnected graph with vertices $u, v$ in different components, then we can define $d(u, v)=\infty$. Hence, for a digraph $D$ that is not strongly connected or a graph $G$ that is disconnected, we define the diameter $\operatorname{diam}(D)$ or $\operatorname{diam}(G)$ to be $\infty$.

For a digraph $D$, the antipodal digraph $A(D)$ of $D$ is the digraph with $V(A(D))=V(D)$ and $E(A(D))=\left\{(u, v) \mid u, v \in V(D)\right.$ and $\left.d_{D}(u, v)=\operatorname{diam}(D)\right\}$. Our first result gives a useful property of antipodal digraphs. The proof is straightforward, so we omit it.

LEMMA 1. If $D$ is a symmetric digraph, then $A(D)$ is also symmetric.

For $1 \leq p(D) \leq 3$, it is easy to check that the converse of Lemma 1 is true. However, 
for $p(D) \geq 4$, there exist asymmetric digraphs with symmetric antipodal digraphs. Figure 1 shows an asymmetric digraph $D_{1}$ of order $p=4$ with $\operatorname{diam}\left(D_{1}\right)=\infty$ and the corresponding symmetric antipodal digraph $A\left(D_{1}\right)$ where the undirected edge between two vertices indicates the presence of both directed arcs between the vertices. Figure 1 shows an asymmetric strong digraph $D_{2}$ of order 4 with finite diameter $\left(\operatorname{diam}\left(D_{2}\right)=3\right)$ and its symmetric antipodal digraph $A\left(D_{2}\right)$.

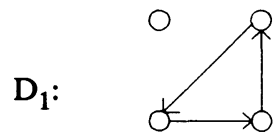
$A\left(D_{1}\right)$ :
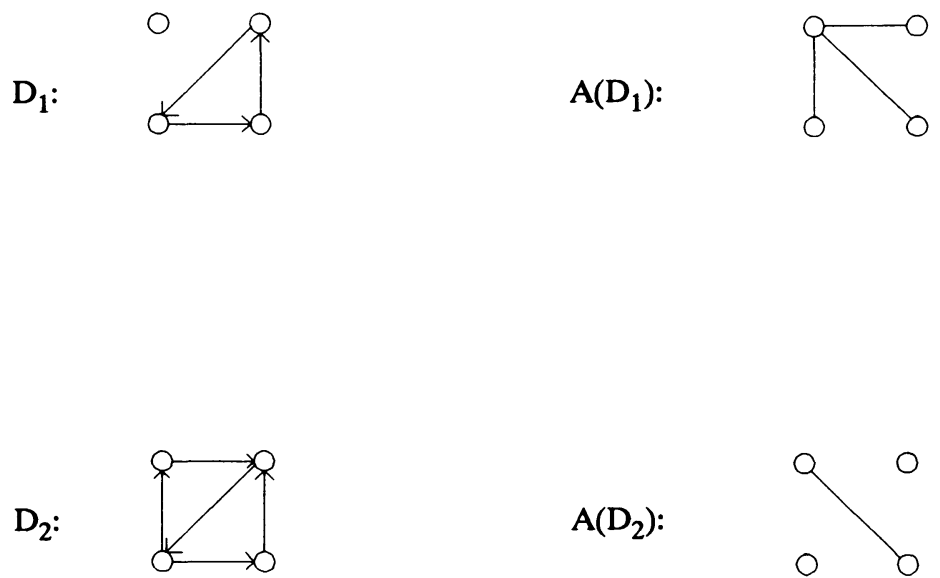
$A\left(D_{2}\right)$ :

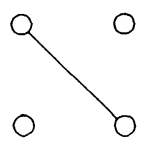

Figure 1

The convention of representing the symmetric pair of $\operatorname{arcs}(u, v)$ and $(v, u)$ by the single edge $u v$ induces a one-to-one correspondence $\varphi$ from the set of symmetric digraphs to the set of graphs. For example, in Figure 1, we have $\varphi\left(A\left(D_{1}\right)\right)=K(1,3)$ and $\varphi\left(A\left(D_{2}\right)\right)=$ $K_{2} \cup \bar{K}_{2}$. Therefore, by Lemma 1 , it is natural to define, for a graph $G$, the antipodal graph $A(G)$ of $G$ as the graph with $V(A(G))=V(G)$ and $E(A(G))=\left\{u v \mid u, v \in V(G)\right.$ and $d_{G}(u, v)=$ $\operatorname{diam}(G)\}$

Antipodal graphs were introduced by Singleton [2] and have been studied by Acharya and Acharya [3], Rajendran [4], Aravamudhan and Rajendran in [5,6], Johns [7], and Chartrand et al. [8] where the following properties have been verified.

PROPOSITION 2. For a graph $G$ of order $p$, the antipodal graph $A(G)=G$ if and only if $G \simeq K_{p}$.

PROPOSITION 3. If $G$ is a non-complete graph of order $p$, then $A(G) \subset \bar{G}$.

PROPOSITION 4. For a graph $G$, the antipodal graph $A(G)=\bar{G}$ if and only if (1) $G$ is of diameter 2 or (2) $G$ is disconnected and the components of $G$ are complete graphs.

In [5] a characterization of antipodal graphs was given which we now state.

PROPOSITION 5. A graph $G$ is an antipodal graph if and only if it is the antipodal graph of its complement. 
Using other results in [5], we can give a second form of Proposition 5.

PROPOSITION 6. A graph $G$ is an antipodal graph if and only if (1) $\operatorname{diam}(\bar{G})=2$ or (2) $\bar{G}$ is disconnected and the components of $\bar{G}$ are complete graphs.

In the next section, we will generalize these results and give a characterization of antipodal digraphs. This will lead to a characterization of antipodal graphs in Proposition 4, our proof of which is simpler than the proof given in [5].

\section{A CHARACTERIZATION OF ANTIPODAL DIGRAPHS.}

We begin with some properties of antipodal digraphs.

LEMMA 7. For a digraph $D$ of order $p$, the antipodal digraph $A(D)=D$ if and only if $D \cong K_{p}^{*}$

PROOF. First, suppose that $A(D)=D$. If $(u, v) \in E(D)$ then $(u, v) \in E(A(D))$. Therefore, $d_{D}(u, v)=1=\operatorname{diam}(D)$. Since $K_{p}^{*}$ is the only digraph of diameter 1 , we have $D \cong K_{p}^{*}$. For the converse, if $D \cong K_{p}^{*}$, then $\operatorname{diam}(D)=1$ and for every pair $u, v$ of vertices in $D$, the distance $d_{D}(u, v)=1$. Hence, $A(D) \cong K_{p}^{*}$ and $A(D)=D$.

Since $\varphi\left(\boldsymbol{K}_{p}^{*}\right)=\boldsymbol{K}_{\boldsymbol{p}}$, Proposition 2 follows immediately.

Suppose that $D * K_{p}^{*}$. Then $\operatorname{diam}(D) \geq 2$ and if $(u, v)$ is an $\operatorname{arc}$ of $D$, then $(u, v)$ will not be an arc of $A(D)$. Similarly, if $(u, v)$ is an arc of $A(D)$, then it is not an arc of $D$. Thus, $A(D)$ is always a sub-digraph of $\bar{D}$. This is our next result.

LEMMA 8. If $D$ is a digraph of order $p$ that is not a complete symmetric digraph, then $A(D) \subset \bar{D}$.

As a special case, we have Proposition 3.

We can now present a result that will be useful in our characterization of antipodal digraphs.

THEOREM 9. For a digraph $D$, the antipodal digraph $A(D)=\bar{D}$ if and only if either (1) $\operatorname{diam}(D)=2$ or (2) $D$ is not strongly connected and for every pair $u, v$ of vertices of $D$, the distance $d_{D}(u, v)=1$ or $d_{D}(u, v)=\infty$.

PROOF. First, suppose that $\operatorname{diam}(D)=2$. If $(u, v) \in E(D)$, then $d_{D}(u, v)=1$; so $(u, v) \notin E(A(D))$. If $(u, v) \notin E(D)$, then, $d_{D}(u, v)=2$ and $(u, v) \in E(A(D))$. Therefore, $A(D)=\bar{D}$. Now, suppose that $D$ is not strongly connected and for every pair $u, v$ of vertices of $D$, the distance $d_{D}(u, v)=1$ or $d_{D}(u, v)=\infty$. If $d_{D}(u, v)=\infty$ for every pair $u, v$ of vertices, then $D \cong \overline{K_{p}^{*}}$ for some positive integer $p$, and $A(D)=A\left(\overline{K_{p}^{*}}\right) \approx K_{p}^{*} \approx \bar{D}$. If, on the other hand, $\operatorname{diam}(D)=\infty$ and $(u, v) \in E(D)$, then $(u, v) \notin E(A(D))$. If $(u, v) \notin E(D)$, then 
$d_{D}(u, v)=\infty$ and so $(u, v) \in E(A(D))$. Hence, $A(D)=\bar{D}$.

For the converse, suppose that $A(D)=\bar{D}$. Assume that the diameter is finite and not equal to 2. If $\operatorname{diam} D=1$, then $D \approx K_{p}^{*}$. However, then $A\left(K_{p}^{*}\right)=\overline{K_{p}^{*}}$, which contradicts Lemma 7. Thus, assume that $2<\operatorname{diam} D<\infty$. Let $u$ and $v$ be vertices of $D$ such that $d_{D}(u, v)=2$. Note that $(u, v) \notin E(D)$ and $(u, v) \notin E(A(D)) ;$ so $A(D) \neq \bar{D}$. Now, assume that $\operatorname{diam} D=\infty$ and there exist vertices $u$ and $v$ such that $1<d_{D}(u, v)<\infty$. Then $(u, v) \notin$ $E(D)$ and $(u, v) \notin E(A(D))$ and again, $A(D) \neq \bar{D}$.

If $D$ is a symmetric digraph of diameter 2 , then $\varphi(D)$ is a graph of diameter 2 . On the other hand, if $D$ is symmetric but not strongly connected and for every pair $u, v$ of vertices of $D$, the distance $d_{D}(u, v)=1$ or $d_{D}(u, v)=\infty$, then $\varphi(D)$ is a disconnected graph where each component is complete. This implies Proposition 4.

We can now give a characterization of antipodal digraphs.

THEOREM 10. A digraph $D$ is an antipodal digraph if and only if $D$ is the antipodal digraph of its complement.

PROOF. First, if $D$ is the antipodal digraph of its complement, then $D$ is an antipodal digraph. For the converse, suppose that $D$ is an antipodal digraph and let $\boldsymbol{H}$ be a digraph such that $A(H)=D$. We consider three cases based on diam $H$.

CASE 1. Suppose that $\operatorname{diam} H=1$. Then $H=K_{p}^{*}$, for some positive integer $p$ and $A(H)=A\left(K_{p}^{*}\right)=K_{p}^{*}-D$. Since $\bar{D} \approx \overline{K_{p}^{*}}$ and $A\left(\overline{K_{p}^{*}}\right)=K_{p}^{*}$, it follows that $D=A(\bar{D})$, as desired.

CASE 2. Suppose that $1<\operatorname{diam} \boldsymbol{H}<\infty$. Since the diameter of $\boldsymbol{H}$ is finite, $\boldsymbol{H}$ is strongly connected and for every pair $u, v$ of vertices of $H$, the distance $d_{H}(u, v) \leq \operatorname{diam} H$. Define $H^{\prime}$ as the digraph formed by adding the arc $(u, v)$ to $E(H)$ if $1<d_{H}(u, v)<\operatorname{diam} H$. Note that $d_{H^{\prime}}(u, v)=1$ when $d_{H}(u, v)<\operatorname{diam}(H)$ and $d_{H^{\prime}}(u, v)=2$ when $d_{H}(u, v)=\operatorname{diam}(H)$. Thus, $D=A(H)=A\left(H^{\prime}\right)$. Since $\operatorname{diam}\left(H^{\prime}\right)=2$, we have $A\left(H^{\prime}\right)=\overline{H^{\prime}}$ by Theorem 9. Therefore, $D=\bar{H}^{\prime}$ and $\bar{D}=H^{\prime}$ which gives $D=A(\bar{D})$, as desired.

CASE 3. Suppose that $\operatorname{diam}(H)=\infty$. Define $H^{\prime}$ as the digraph formed by adding the arc $(u, v)$ to $E(H)$ if $1<d_{H}(u, v)<\operatorname{diam}(H)$. Now, if $d_{H}(u, v)<\infty$, then $d_{H^{\prime}}(u, v)=1$ and if $d_{H}(u, v)=\infty$, then $d_{H^{\prime}}(u, v)=\infty$ also. Thus, $D=A(H)=A\left(H^{\prime}\right)$. Since $H^{\prime}$ is not strongly connected and for every pair $u, v$ of vertices of $H^{\prime}$ the distance $d_{H^{\prime}}(u, v)=1$ or $d_{H^{\prime}}(u, v)=1$ we have $A\left(H^{\prime}\right)=\overline{H^{\prime}}$ by Theorem 9. Therefore, $D=\overline{H^{\prime}}$ and $\bar{D}=H^{\prime}$ which gives $D=A(\bar{D})$, as desired. 
This characterization can be restated, with the aid of Theorem 9, as follows.

COROLLARY 11. A digraph $D$ is an antipodal digraph if and only if (1) $\operatorname{diam}(\bar{D})=2$ or (2) $\bar{D}$ is not strongly connected and for every pair $u, v$ of vertices of $D$, the distance $d_{\bar{D}}(u, v)=1$ or $d_{\bar{D}}(u, v)=\infty$.

With the correspondence $\varphi$ between symmetric digraphs and graphs, the characterizations of antipodal graphs in Proposition 5 and Proposition 6 follow immediately.

\section{-3. SELF-ANTIPODAL DIGRAPHS AND GRAPHS.}

In the previous section, we proved, for a digraph $D$ of order $p$, that the antipodal digraph $A(D)$ is identical to $D$ if and only if $D \simeq K_{p}^{*}$. Similarly, for a graph $G$ of order $p$, the antipodal graph $A(G)$ is identical to $G$ if and only if $G \approx K_{p}$. A more interesting question can also be asked. When is $A(D)$ isomorphic to $D$ or when is $A(G)$ isomorphic to $G$ ? If $A(D) \approx D$, then we will call $D$ a self-antipodal digraph and if $A(G) \approx G$, we will call $\boldsymbol{G}$ a self-antipodal graph. Self-antipodal graphs were studied in [3].

Although no characterization is known for self-antipodal digraphs, certain types of digraphs are known to be self-antipodal. First, the complete symmetric digraphs are self antipodal. In addition, given a positive integer $p \geq 3$, the directed cycle $C_{p}^{\prime}$, where $V\left(C_{p}^{\prime}\right)=$ $\left\{v_{1}, v_{2}, \ldots, v_{p}\right\}$ and $E\left(C_{p}^{\prime}\right)=\left\{\left(v_{i}, v_{i+1}\right) \mid 1 \leq i \leq p-1\right\} \cup\left\{\left(v_{p}, v_{1}\right)\right\}$, is self-antipodal. Both of these classes of digraphs are strongly connected; however, there exist self-antipodal digraphs that are not strongly connected. The self-antipodal digraph $D$ in Figure 2 is an example of minimum order that is weakly connected but not unilaterally connected.

For a class of self-antipodal digraphs $D$ that are unilaterally connected but not strongly connected, let $p>1$ be an integer and let $D=T_{p}$, the transitive tournament of order $p$. Note that if $(u, v) \in E\left(T_{p}\right)$, then $d_{T_{p}}(v, u)=\infty$. Thus, $o d_{A\left(T_{p}\right)}(v)=i d_{T_{p}}(v)$ and since the sequence of indegrees of the vertices of $T_{p}$ is $0,1,2, \ldots, p-1$ we have the same sequence as the score sequence for $A\left(T_{p}\right)$. Because $T_{p}$ is the only tournament with score sequence $0,1, \ldots, p-1$, it follows that $A\left(T_{p}\right) \cong T_{p}$.

If $D$ is a disconnected digraph, then $D$ is not self-antipodal because $A(D)$ is strongly connected and $\operatorname{diam} A(D) \leq 2$. To see this, let $u$ and $v$ be vertices of $D$. If $u$ and $v$ are in different components of $D$, then $d_{D}(u, v)=\infty=\operatorname{diam}(D)$. Thus $(u, v) \in E(A(D))$ and $d_{A(D)}(u, v)=1$. If, on the other hand, $u$ and $v$ are in the same component of $D$, then there exists a vertex $w$ in a second component of $D$. Now, $d_{D}(u, w)=d_{D}(w, v)=\infty$, so $(u, w) \epsilon$ $E(A(D))$ and $(w, v) \in E(A(D))$ and $d_{A(D)}(u, v) \leq 2$. Therefore $A(D)$ is strongly connected and $\operatorname{diam}(A(D)) \leq 2$. 
D:

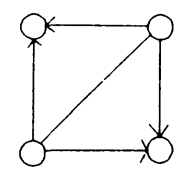

Figure 2

Also, $D$ is not self-antipodal if $D$ is strongly connected and the eccentricity of some vertex $v$ of $D$ is less than the diameter of $D$. In this case, $\operatorname{od}_{A(D)}(v)=0$ and $A(D)$ is not strong.

We combine these two observations in the next result.

LEMMA 12. If $D$ is a self-antipodal digraph, then $D$ is weakly connected. If, in addition, $D$ is strongly connected, then $D$ is self-centered.

An immediate consequence of Theorem 9 and Lemma 12 is as follows.

THEOREM 13. If $D$ is a self-complementary digraph of order $p \geq 2$, then $A(D)=D$ if and only if (1) diam $(D)=2$ or (2) $D$ is not strongly connected and for every pair $u, v$ of vertices of $D$, the distance $d_{D}(u, v)=1$ or $d_{D}(u, v)=\infty$.

We now present a result on the size of self-antipodal digraphs.

THEOREM 14. If $D$ is a non-complete self-antipodal digraph of order $p \geq 3$, then $p \leq q(D) \leq p(p-1) / 2$.

PROOF. By Lemma 12, the digraph $D$ must be weakly connected. The minimum number of arcs in a weakly connected digraph is $p-1$, so $p-1 \leq q(D)$. Suppose that $q(D)=p-1$. Then $D$ can contain no directed cycles and hence $D$ is not strongly connected. If $D$ is unilaterally connected, then $D$ contains a directed walk that passes through each vertex of $D$. This can only be done with $p-1$ arcs if $D$ is a directed path $P^{\prime}$. However, since $A\left(P^{\prime}\right)$ is isomorphic to a transitive tournament, $D$ is not self-antípodal. Finally, if $D$ is weakly connected, but not unilaterally connected, then there exist two vertices $u$ and $v$ in $D$ such that no $u-v$ directed path and no $v-u$ directed path exist in $D$. Therefore, $d_{D}(u, v)=$ $d_{D}(v, u)=\infty$ and the $\operatorname{arcs}(u, v)$ and $(v, u)$ are both in $A(D)$. Since $D$ contains no directed cycles and $A(D)$ contains a directed 2-cycle, $A(D) * D$. Therefore, $q(D) \geq p$.

For the upper bound, we know since $D * K_{p}^{*}$ that $A(D) \subset \bar{D}$. Now $D=A(D) \subset \bar{D}$ implies that $q(D) \leq q(\bar{D})$. Therefore, $q(D) \leq \frac{1}{2} q\left(K_{p}^{*}\right)=p(p-1) / 2$.

The bounds in Theorem 14 are sharp. The lower bound is sharp for the class of directed cycles and the upper bound is sharp for the class of transitive tournaments. It is not true, however, for given positive integers $p$ and $c$ with $p<c<p(p-1) / 2$, that there is a selfantipodal digraph $D$ having $q(D)=c$. For instance, there is no self-antipodal digraph with four vertices and five arcs. 
We now turn to self-antipodal graphs. If $G$ is a self-antipodal graph and $\varphi$ is the natural one-to-one correspondence from the set of symmetric digraphs to the set of graphs, then $\varphi^{-1}(G)$ is a self-antipodal digraph. By Lemma 12 , the digraph $\varphi^{-1}(G)$ is weakly connected; so $G$ is connected. Also, since $\varphi^{-1}(G)$ is symmetric, it is strongly connected and thus $\varphi^{-1}(G)$ and $G$ are self-centered. Therefore, we have the following result.

THEOREM 15. If $G$ is a self-antipodal graph, then $G$ is connected and self-centered.

Now, suppose that $\boldsymbol{G}$ is a self-antipodal self-complementary graph of order $p \geq 2$. By Theorem 15, the graph $\boldsymbol{G}$ is connected and so Proposition 4 implies that $A(G)=\bar{G} \approx G$ if and only if $\boldsymbol{G}$ has diameter 2 . We state this as the next result.

COROLLARY 16. Let $\boldsymbol{G}$ be a self-complementary graph of order $\dot{p} \geq 2$. Then $G$ is $a$ self-antipodal graph if and only if diam $G=2$.

An important class of self-antipodal graphs is the class of odd cycles. To see this, we will show that $A\left(C_{2 d+1}\right), d \geq 1$, is 2-regular and connected - a characterization of cycles. First, let $V\left(C_{2 d+1}\right)=\left\{v_{1}, v_{2}, \ldots, v_{2 d+1}\right\}$ and $E\left(C_{2 d+1}\right)=\left\{v_{l} v_{i+1} \mid 1 \leq i \leq 2 d\right\} U\left\{v_{2 d+1} v_{1}\right\}$. Now, $\operatorname{diam}\left(C_{2 d+1}\right)=d$ and for each vertex $v_{i}, 1 \leq i \leq 2 d+1$, there exist exactly two vertices $v_{j}$ and $v_{k}$ with $j \equiv(i+d) \bmod (2 d+1)$ and $k \equiv(i+d+1) \bmod (2 d+1)$ such that $d\left(v_{i}, v_{j}\right)=d\left(v_{i}, v_{k}\right)=d$. Thus, $v_{i}$ is adjacent to only $v_{j}$ and $v_{k}$ in $A\left(C_{2 d+1}\right)$, and so $A\left(C_{2 d+1}\right)$ is 2-regular. Next, if $u$ and $v$ are distinct vertices in $C_{2 d+1}$, then, without loss of generality, we can let $u=v_{2 d+1}$ and $v=v_{n}$ with $1 \leq n \leq d$. A $u-v$ path in $A\left(C_{2 d+1}\right)$ is $P: u=v_{2 d+1}, v_{d+1}, v_{1}, v_{d+2}, v_{2}, \ldots, v_{d+n}, v_{n}$, so $A\left(C_{2 d+1}\right)$ is connected and it follows that $A\left(C_{2 d+1}\right) \approx C_{2 d+1}$.

We will now define a class of self-antipodal graphs of even order.

For $d \geq 3$, let $G$ be the graph of order $2 d+2$ and size $2 d+3$ such that $V(G)=$ $\left\{v_{0}, v_{1}, \ldots, v_{2 d+1}\right\}$ and $E(G)=\left\{v_{i} v_{i+1} \mid 1 \leq i \leq 2 d\right\} U\left\{v_{2 d+1} v_{1}, v_{2 d+1} v_{0}, v_{0} v_{2}\right\}$. Vertices $v_{0}$ and $v_{1}$ are similar and $G-v_{0} \cong C_{2 d+1}$. For vertices $u$ and $v$ in $G-v_{0}$, the distance $d_{G-v_{0}}(u, v)=d_{G}(u, v)$. Thus, $A\left(G-v_{0}\right)$ is an induced subgraph of $A(G)$. Now $A\left(G-v_{0}\right)=$ $C_{2 d+1}$ with $v_{1}$ adjacent to $v_{d+1}$ and $v_{d+2}$. Since $v_{0}$ and $v_{1}$ are similar in $G$, the vertex $v_{0}$ is also adjacent only to $v_{d+1}$ and $v_{d+2}$ in $A(G)$ and hence, $A(G) \approx G$. We will henceforth denote this graph by $G(2 d+2)$.

We now present a necessary condition for the size of self-antipodal graphs.

THEOREM 17. Let $G$ be a non-complete self-antipodal graph of order $p \geq 5$. Then

(1) $p \leq q(G) \leq\lfloor p(p-1) / 4\rfloor$, if $p$ is odd, and

(2) $p+1 \leq q(G) \leq\lfloor p(p-1) / 4\rfloor$, if $p$ is even.

PROOF. We first show that $\lfloor p(p-1) / 4\rfloor$ is an upper bound. The antipodal graph $A(G) \subset \bar{G}$ implies that $G \subset \bar{G}$; so $q(G) \leq\left\lfloor\frac{1}{2} q\left(K_{p}\right)\right\rfloor=\lfloor p(p-1) / 4\rfloor$. 
Now consider the lower bounds. By Theorem 15, we know that $G$ is connected. Hence, $p-1 \leq q(G)$. However, if $q(G)=p-1$, then $G$ is a tree and $G$ is not selfcentered. This is because if $u$ is an end-vertex of $G$ and $v$ is the vertex adjacent to $u$, then $e(u)=e(v)+1$. Thus, $p \leq q(G)$. Now suppose that $p$ is even and there exists a selfantipodal graph $\boldsymbol{G}$ of order $\boldsymbol{p}$. Then $\boldsymbol{G}$ is connected and contains one cycle. Remove one cycle edge $e$ from $G$ to form the tree $G-e$ and let $V$ be the set of end-vertices of $G-e$. Suppose that there exists a vertex $u \in V$ such that $u$ is not incident with $e$ in $G$. Then, if $v$ is the vertex adjacent to $u$ in $G$, then $e(u)=e(v)+1$. Hence, $G$ is not self-centered and therefore, not self-antipodal. On the other hand, suppose that every vertex of $V$ is incident with the edge $e$. Then $|V|=2$ and $G=C_{p}$. However, for $p$ even, $A\left(C_{p}\right)=\left(\frac{p}{2}\right) K_{2}$ and again $\boldsymbol{G}$ is not self-antipodal.

Therefore, if $p \geq 5$ is odd, then $p \leq q(G) \leq\lfloor p(p-1) / 4\rfloor$ and if $p>5$ is even, then $p+1 \leq q(G) \leq\lfloor p(p-1) / 4\rfloor$.

The bounds in Theorem 17 are sharp. First, for each odd integer $p \geq 5$ the cycle $C_{p}$ is self-antipodal and $q\left(C_{p}\right)=p$. For each even integer $p \geq 8$, the graph $G(p)$ is self-antipodal and $q(G(p))=p+1$. Second, for each positive integer $p \geq 5$ such that $p=0(\bmod 4)$ or $P \equiv 1(\bmod 4)$, there exists a self-complementary graph $G$ of diameter 2 . By Corollary 16, the graph $G$ is self-antipodal. Hence, $q(G)=q(\bar{G})=\frac{1}{2} q\left(K_{p}\right)=p(p-1) / 4$.

Two questions still remain. Do there exist self-antipodal graphs of order $p \equiv 2(\bmod 4)$ or $p \equiv 3(\bmod 4)$ and size $\lfloor p(p-1) / 4\rfloor$ ? Also, for which positive integers $p \geq 5$ and $c$ such that $p \leq c \leq\lfloor p(p-1) / 4\rfloor$, does there exist a self-antipodal graph $G$ with $c=q(G)$ ?

\section{REFERENCES}

1. CHARTRAND, G. and LESNIAK, L. Graphs and Digraphs, 2nd Edition, Wadsworth and Brooks/Cole, Monterey, CA, 1986.

2. SINGLETON, R. There Is No Irregular Moore Graph, Amer. Math. Monthly 75 (1968), 42-43.

3. ACHARYA, B.D. and ACHARYA, M. On Self-antipodal Graphs, Nat. Acad. Sci. Lett. 8 (1985), 151-153.

4. RAJENDRAN, B. Topics In Graph Theory: Antipodal Graphs, Ph.D. thesis, 1985.

5. ARAVAMUDHAN, R. and RAJENDRAN, B. On Antipodal Graphs, Discrete Math. 49 (1984), 193-195.

6. . ARAVAMUDHAN, R. and RAJENDRAN, B. A Note On "On Antipodal Graphs," Discrete Math. 58 (1986), 303-305.

7. JOHNS, G. Generalized Distance In Graphs, Ph.D. thesis, 1988.

8. CHARTRAND, G., JOHNS, G. and OELLERMANN, O.R. On Peripheral Vertices In Graphs, Topics in Combinatorics and Graph Theory, Physica-Verlag Heidelberg (1990), 193-199. 


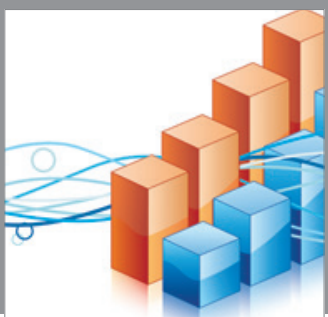

Advances in

Operations Research

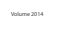

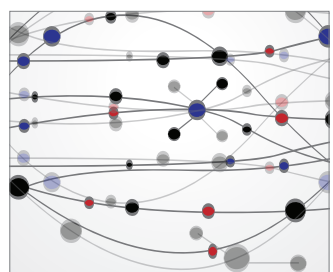

\section{The Scientific} World Journal
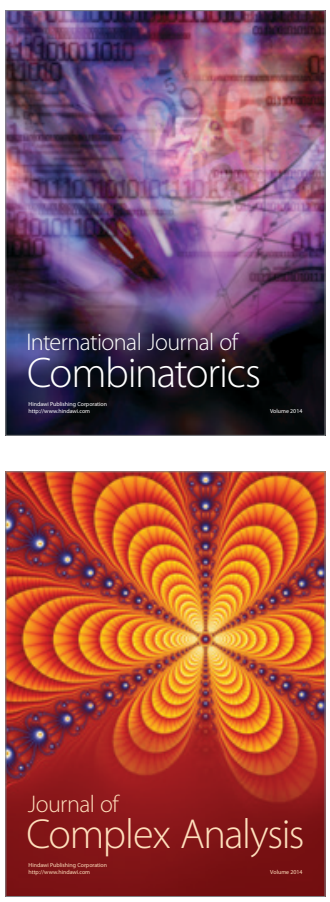

International Journal of

Mathematics and

Mathematical

Sciences
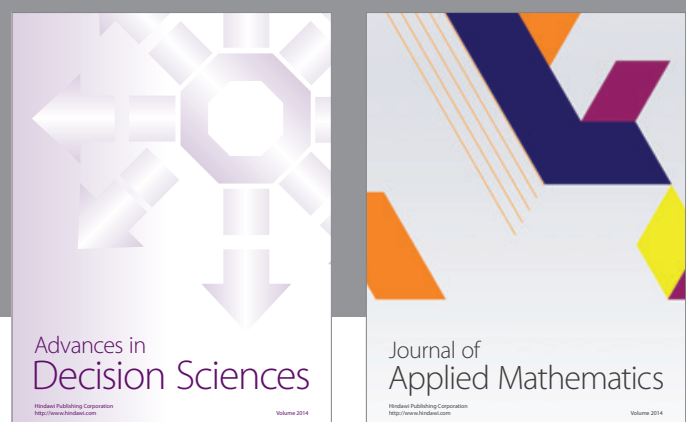

Journal of

Applied Mathematics
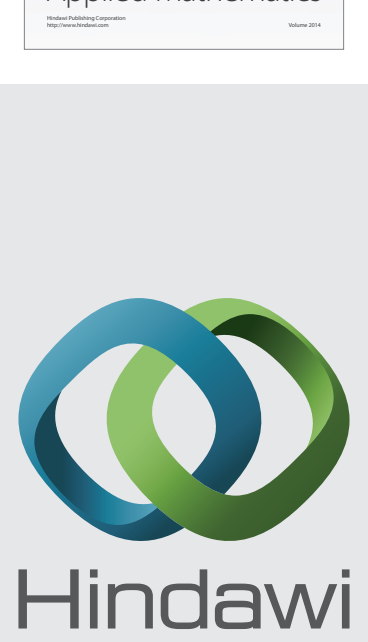

Submit your manuscripts at http://www.hindawi.com
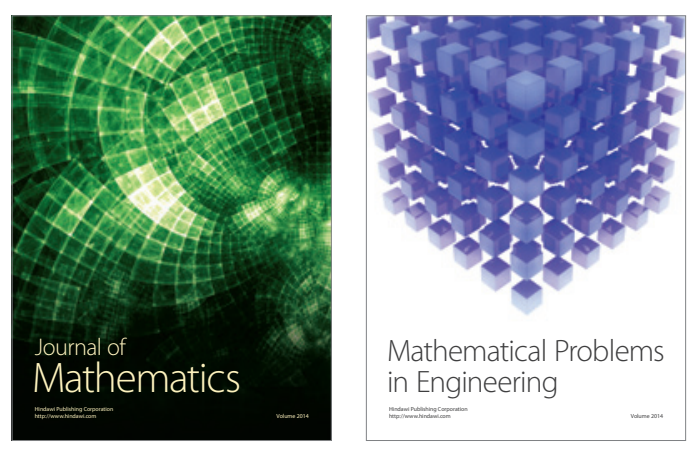

Mathematical Problems in Engineering
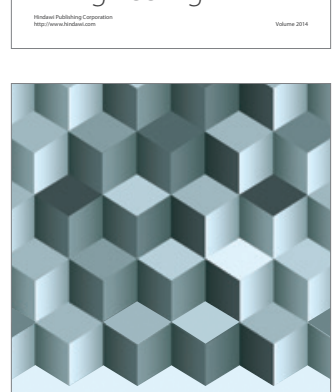

Journal of

Function Spaces
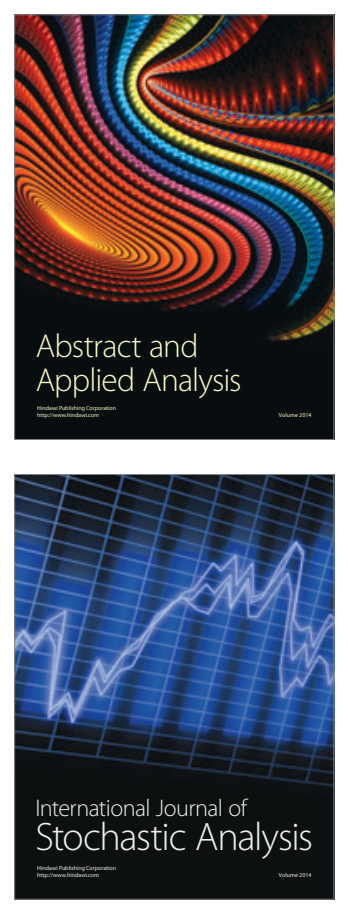

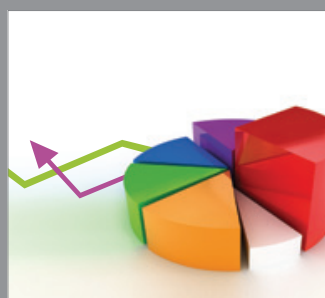

ournal of

Probability and Statistics

Promensencen
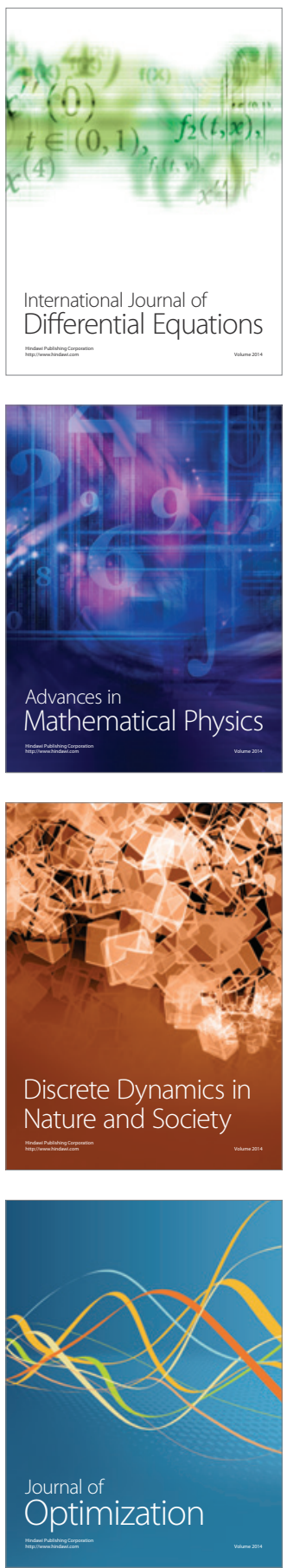